\title{
Towards Immersive and Social Audience Experience in Remote VR Opera
}

\author{
Alina Striner \\ DIS CWI \\ Netherlands \\ alina.striner@cwi.nl \\ Thomas Röggla \\ Centrum Wiskunde \& Informatica \\ Netherlands \\ t.roggla@cwi.nl
}

\author{
Sarah Halpin \\ Irish National Opera \\ Ireland \\ sarah@irishnationalopera.ie \\ Pablo Cesar \\ CWI \\ Netherlands \\ p.s.cesar@cwi.nl
}

\begin{abstract}
Opera is a historic art that struggles to be approachable to modern audiences. In partnership with the Irish National Opera (INO), this work considers how VR may be used to develop a new form of immersive opera. To this end, we ran three open-ended focus groups to consider how creative, multisensory, and social VR technology may be employed in digital opera. Our findings assert the importance of creating an immersive experience by safely giving audiences agency to interact, to democratize personal and social experiences, and to consider different ways of representing their bodies, their social rituals, and the virtual social space. Using these findings, we envision a new form of VR opera that couples physical traditions with digital affordances.
\end{abstract}

\section{CCS CONCEPTS}

- Human-centered computing $\rightarrow$ Virtual reality; Collaborative interaction.

\section{KEYWORDS}

virtual reality; opera

ACM Reference Format:

Alina Striner, Sarah Halpin, Thomas Röggla, and Pablo Cesar. 2021. Towards Immersive and Social Audience Experience in Remote VR Opera. In ACM International Conference on Interactive Media Experiences (IMX '21), June 21-23, 2021, Virtual Event, NY, USA. ACM, New York, NY, USA, 8 pages. https://doi.org/10.1145/3452918.3465490

\section{INTRODUCTION}

Opera as an art form spans several centuries of culture and language. Although rich in tradition, contemporary opera companies struggle to convey its relevance to modern audiences, and to overcome legacy preconceptions of the form as overly expensive, stuffy, and unwelcoming [34]. To bring in new audiences, opera companies have begun cinema simulcasts and performance web streams [31];

Permission to make digital or hard copies of part or all of this work for personal or classroom use is granted without fee provided that copies are not made or distributed for profit or commercial advantage and that copies bear this notice and the full citation on the first page. Copyrights for third-party components of this work must be honored

For all other uses, contact the owner/author(s).

IMX '21, June 21-23, 2021, Virtual Event, NY, USA

(c) 2021 Copyright held by the owner/author(s).

ACM ISBN 978-1-4503-8389-9/21/06.

https://doi.org/10.1145/3452918.3465490 these services make the content broadcasting more accessible, but do not solve the problem of making the content representation approachable.

Over the last fifty years, media consumption has transitioned from a passive into an interactive format, and from a social experience into a personal one, with the power to immerse and empower audiences across domains [39]. In particular, virtual reality (VR) researchers and designers have begun to digitally recreate the physical experience of the real world [14,33], showing the value of sensory realism for storytelling [18], emotion, and presence [9]. In Hamlet on the Holodeck, Murray [32] extends VR into an interactive social experience, akin to live theater, that transforms passive readers into audiences engaged in immersive and reactive narratives [32]. Digital artists and designers across storytelling and entertainment domains have responded to this need for immersion with a range of virtual and augmented experiences that support a spectrum of audience experiences [39].

This work considers how VR may be used to transform traditional opera into an immersive, interactive, and social experience. In partnership with the Irish National Opera (INO), we ran three open-ended focus groups to consider how creative, multisensory, and social VR technology may be use to develop a new form of immersive opera. First, we contextualize our work in literature immersion through sensory immersion, audience participation, and social experience. After this, we describe our focus group method and present our results. Our findings assert the importance of creating an immersive experience by safely giving audiences agency to interact, to democratize personal and social experiences, and to consider different ways of representing their bodies, their social rituals, and the virtual social space. Using these findings, we envision a new form of opera that couples physical traditions with digital affordances.

\section{RELATED WORK}

The following sections describe our related work. First, we introduce the concept of immersion. Then, we describe how immersion is enacted through sensory engagement, audience participation, and social experience. 


\subsection{Immersion}

In literature, immersion has been descried as a feeling of "deep play" $[7,11]$ based on engagement, and the intensity and emotional quality of user involvement $[13,15]$. Engagement-based immersion has been shown to activate emotional investment and sustained cognitive involvement [3, 13, 40]. Ermi and Mayra [11] divide the concept of immersion into sensory immersion, task immersion, and imaginative immersion.

2.1.1 Presence through Sensory Immersion. Brockmyer [3] suggests that sensory immersion often creates a sense of presence in an environment, the feeling of "being there," in another reality that takes over attention and perception [7, 11,30]. Researchers and designers have considered may ways to create immersion through sensory engagement. Heilig patented the multisensory Sensorama machine as early as 1962 [12], and recently research has shifted technology away from disembodied interactions toward "realitybased" experiences [19]. In VR, researchers have employed sensory immersion to create presence in entertainment experiences. For instance, Ambioterm [35] and Amphibian [20] simulated environmental conditions in a VR headset, and Martins [28] conceptualized multisensory VR wine tourism.

2.1.2 Task Immersion through Audience Participation. In complement to sensory immersion, Csikszentmihalyi and others $[6,10,11$, $22,36]$ introduce the concept of flow, a state of total task immersion. In entertainment experiences, task immersion has emerged through audience participation, empowering audiences beyond traditional roles [39], and shifting spectatorship from passive to active participation [37, 41]. Reviewing theater, theme park, and game literature, Striner [39] defines audience interactivity as a range of experiences and roles that may allow audiences to participate or interact, and describes a spectrum of audience interactivity in entertainment. This include personalizing an experience, reacting to performance, influencing and augmenting an experience, bidirectionally influencing performers, becoming a performer, and even taking over a performance experience.

2.1.3 Immersion through Social Experience. Beyond entertainment, theater literature underscores the importance of the communal experience to the practice of theater-going [24, 42]. Music performances stimulate social interactions among listeners, allowing friends to deepen their knowledge about each other's music preferences and choices [25]. In opera, Quinn further describes the symbolic social ritual of dressing up and attending a performance [34].

HCI likewise demonstrates the importance of social interaction to the larger immersion and interaction experience $[4,16$, 17]. For instance, game live-streaming describes authentic bidirectional connections between streamers and audiences akin to "seeing friends" [44]. Online environments may even act as virtual "third places" [44], communities with a multidimensional ecosystem of relationships between streamers and viewers [43]. Such rich interaction has been shown to increase viewer attitudes toward virtual performance experiences and enhance their perceived value [5].

\section{METHOD}

We held three (3) focus groups to explore creative, multisensory, and social VR technology. The goals of these focus groups were
1) to understand artist attitudes toward VR as a tool for audience engagement, 2) explore new approaches to enhance user experience through VR, and 3) inform initial user requirements for an immersive VR experience. The following section describes the participant selection process, focus group structure, and our qualitative analysis method.

\subsection{Participant Selection}

Five (5) theater and technology experts participated in the focus groups. All participants had substantial experience and exposure to VR as artistic creators or audience members. The range of participant expertise allowed us to have a lively and informed dialogue with a depth of insight from contrasting perspectives.

They included:

- Director of the INO VR community opera

- Producer of the INO VR community opera

- Artistic director of the Civic Theatre in Tallaght, a community partner working with INO

- Postdoctoral Research Fellow at V-SENSE Project, Trinity College Dublin

- Postdoctoral Research Fellow at V-SENSE Project, Trinity College Dublin

In addition to the the core participants, we invited eleven (11) project partners to observe. These observers took notes, and had an opportunity to ask questions and make observations at the end of each focus group.

\subsection{Session Overview}

We conducted the three focus groups over a one-day virtual Zoom call. Two moderators led the focus groups; a digital communications manager at INO, and a researcher on immersive entertainment technology. The first moderator led the focus groups. The second moderator took notes, then summarized key discussion points at the end of each focus group.

The day was divided into three distinct focus group topics: (1) operatic and creative $V R$ content, (2) sensory enhancements in $V R$ and (3) social VR. The first focus group considered ways to create operatic content in VR. Shown in figure 1, the first presentation considered VR examples across the performing arts, and introduced participants and observers to motion capture technology. Session 2 focused on multisensory enhancements to VR experiences, considering gestural and touch inputs, as well as haptic and scent outputs. Finally, session 3 focused on social VR. First, it described distributed theater environments (figure 1), then considered ways of representing audiences, as avatars, or through volumetric video, in line with work by Jansen [21] and Li [26].

\subsection{Focus Group Structure}

Each focus group consisted of four parts:

- A 20-minute presentation on the topic by one of the project technology teams

- A 45-minute focus group session

- A 15 minute session for project partners to ask questions or make observations 
- A 30 minute session to summarize and receive feedback on key points

The presentations acted as seeds of inspiration for the focus group discussions. After each presentation, the first moderator guided participants through a discussion employing a set of guiding questions (Table 1), developed with INO to provoke a broad discussion on the three subjects. Each session finished with an opportunity for the observers to add perspectives or gain clarity on the discussion. After this, the second moderator summarized the key discussion points, and agreed on a set of conclusions with focus group participants. The summary and conclusion sections helped moderators distill key elements of each discussion, and allowed participants and observers to connect each section to larger themes that had been brought up throughout the day.

\subsection{Debrief}

The moderators created a debrief document using their notes paired with the focus group summaries and conclusions. This process extracted a number of questions and challenges for further research and exploration. The debrief document was shared with the focus group participants for input and comment.

\subsection{Qualitative Analysis}

The three focus groups were analysed using thematic analysis, a qualitative method for identifying, analysing, and reporting themes that is more flexible than traditional grounded theory [2]. We employed a modified version of the six-phase framework developed by Braun and Clarke $(2006)[1,27]$ to identify important themes and sub-themes.

The two moderators conducted the thematic analysis together. The second moderator completed the coding process, a practice inline with standard HCI qualitative methods [29]. When employing one coder, qualitative findings are often triangulated by reviewing results with other members of the community. For this process, the debrief document was paired with transcripts of focus groups. First, the second moderator created a codebook from the debrief document. This codebook integrated the key points of the three focus groups into one set of codes. They coded the audio recording transcripts using this codebook.

After transcription, the moderator coded the dataset using the debrief codes, by reading through the focus group transcriptions multiple times, looking for key points about audience experience, and adding appropriate codes. For points that were not adequately expressed by the data set, the moderator updated the codebook with new codes that represented these points, and re-coded the dataset for these new codes. Likewise, when existing codes did not adequately reflect the nature of the data, the moderator renamed the codes and descriptions, and re-coded the dataset, reconsidering how the dataset represented the differences in code names.

After iteratively coding the dataset, the moderators met to discuss the names and descriptions of the codes, and made notes on any codes that seemed inconsistent with the second coder's experience. They reworked the codes based on this feedback, and made a final pass through the dataset, updating codes based on the final version of the codebook. A set of samples codes are shown in figure 2. After this, the two moderators generated high-level themes together. The second moderator coded the dataset for these themes, and wrote up the results based on the final set of themes and codes, validating the representation of the data with two project partners that observed the focus groups.

\section{RESULTS}

This section presents the themes from the three focus groups: 1) immersion, 2) agency, 3) democratization, and 4) virtual representation. We describe each theme and related codes, and demonstrate their relationship through relevant quotes.

\subsection{Immersion}

We found that immersion was essential to the audience experience; it could evoke emotional and transformative experiences, but was moderated by technology fidelity. During the focus groups, participants described immersion that came from feeling present inside the frame of VR, and created empathy and emotional connection. "You can be in somebody else's shoes and actually feel... what a person should be feeling, probably tapping into some mirror in your own because of the spatial nature of the medium," one participant explained. Another described the visceral experience of watching a lion hunt in VR. "I was sat on the edge of the crevice with this extraordinary tornado of wildebeest, rushing three feet away from me, just below my line and about three feet away from me lying on the top of the side and nonchalant way was a female lion watching lunch go by." As one participant demonstrated, such immersion could even transformative. In a Burning Man VR experience, they described feeling "like I was twirling batons... [like] I took some drug...I was taking masks and put them on...I learned how to fly... within seconds, I was transformed into all the beast in me."

Although it had the potential to be powerful, this immersion was tempered by the fidelity of an experience. Audiences know what the real world looks like, a participant explained. "They won't forgive those glitches because they know them." Likewise, participants had mixed views on the effectiveness of sensory immersion on VR; sensory technology could link participants to to their own memories of an experience, but could be disturbing if it did not map correctly. "By the time [a video] is shown on screen, the smell should already be in the air," a participant related. "But if you throw it exactly at the same time that it's showing on screen, it might take some time to reach the user."

\subsection{Agency}

Agency was likewise intrinsic to the audience experience in VR. A participant felt that designers should consider if the audience "has some agency to take a decision...that links to non-linear storytelling...if the viewer medium gives us a space that we can show things in multi-layers, then that's something that should be exploited." In complement, focus groups participants expressed the importance of personalization, and considered how to create a feedback loop mechanism between audience members and the show. To support the experience of agency, participants also related the importance of designing technology for intuitive gestures.

Creating a non-judgmental play space was also key to audience participation. During the focus groups, participants discussed the importance of developing trust by allowing audiences to say things 


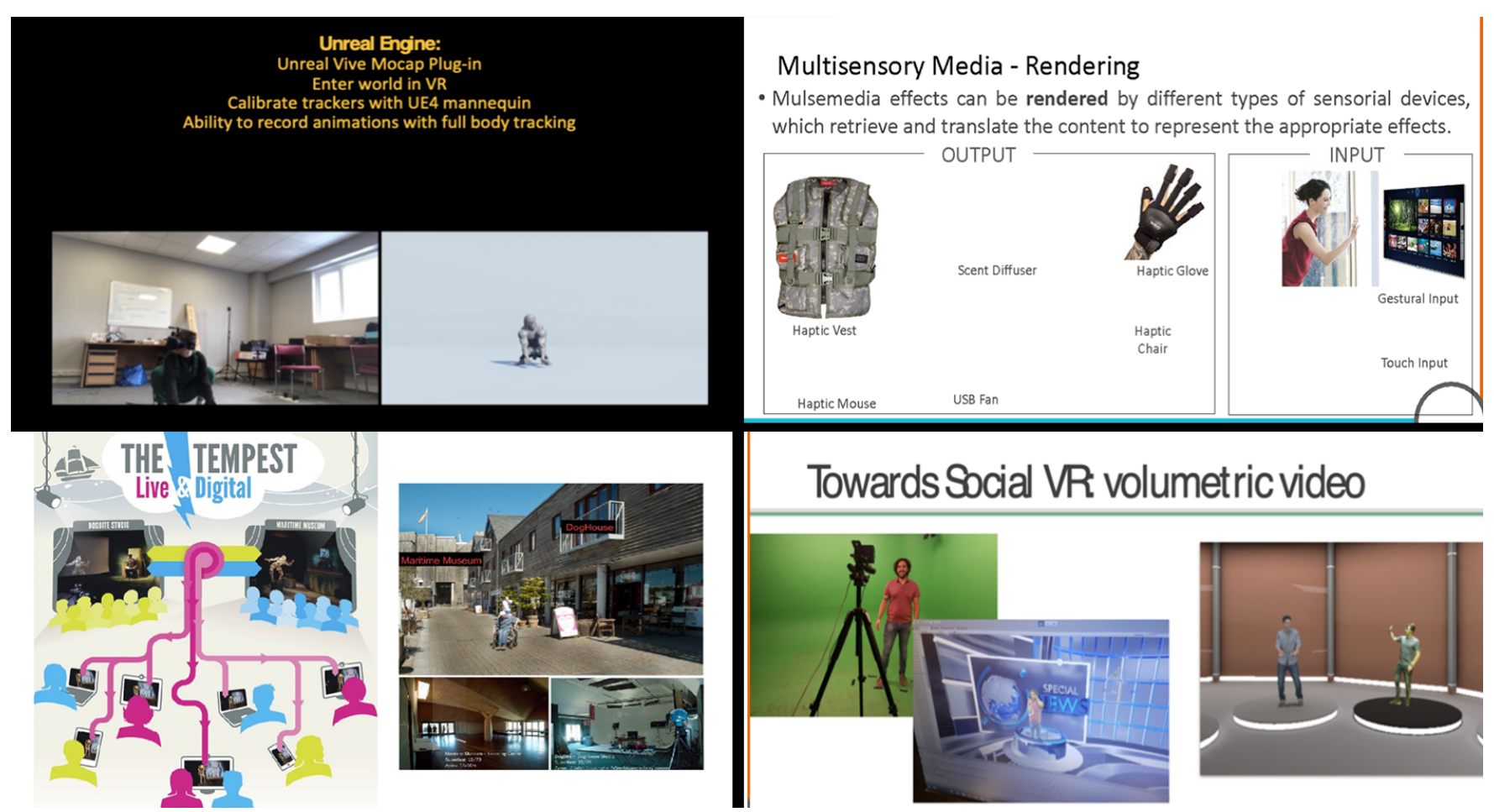

Figure 1: Slides from the three focus groups. Top Left: Slide from the first presentation showing motion capture technology. Top Right: Slide from second presentation on multisensory media technology. Bottom Left: Slide from the third presentation on remote performance experiences. Bottom Right: Slide from the third presentation on volumetric video.

Table 1: Guiding questions for Focus Groups 1, 2 and 3.

Table 1: Guiding questions for Focus Groups 1,2 and 3.
Fuiding Questions
1: VR Content
(1) What experiences have you had with VR? Go around the group, say your name, your involvement
with the project, and describe one memorable experience
(2) What do you think VR can add to the artistic experience?
(3) What are some challenges to using VR for artistic experiences? Any accessibility concerns?

2: Multisensory Media

(1) How might multisensory technology (haptics, other?) enhance the experience of opera?

(2) What multisensory experiences would you like to see integrated into an operatic VR piece?

(3) How might multisensory experiences be disturbing?

3: Social VR

(1) How might social VR technology enhance the audience's experience of opera? In what way?

(2) What kind of social VR experiences would you like to see in a VR opera?

(3) What are some challenges audiences could have using social VR when viewing an opera?

that may be unusual in normal life. It was important to "have a closed network where people felt that it was a safe space for them to interact with each other, and they'd have to have some source of knowledge of what to expect when they arrived." To do this, focus group participants disclosed the importance of moderating the experience, and accounting for trolling. Still, participants acknowledged that not all experiences would appeal to all audience members.

\subsection{Democratization}

The third theme, democratization, considered different ways to support VR opera through experience and social democratization. Participants discussed the importance of experience democratization for audience members through onboarding and accessibility considerations. In the Social VR focus group, participants asked how to create an effective gateway context for a virtual show experience, and suggested an engagement process that began with buying a ticket. The experience begins "the moment that you actually buy 


\begin{tabular}{|c|c|c|}
\hline Virtual Representation & Body Representation & $\begin{array}{l}\text { "if I'm going to the opera with my wife...we have certain gestures, certain } \\
\text { things that we understand...that an avatar is never going to [be able to] do" }\end{array}$ \\
\hline Virtual Representation & $\begin{array}{l}\text { Ritual/Ceremony } \\
\text { Representation }\end{array}$ & $\begin{array}{l}\text { "although we go to the theater,...for the performance...we're also going for, } \\
\text { um, for the idea of dressing up, you know, just showing, you know, showing } \\
\text { ourselves off to one another." }\end{array}$ \\
\hline Virtual Representation & $\begin{array}{l}\text { Social Space } \\
\text { Representation }\end{array}$ & $\begin{array}{l}\text { "you could just...leave...during the installation when you're bored. There's } \\
\text { people that you talk to, um, at the beginning, and maybe sometimes you go } \\
\text { back to the smoking area and that's how you meet someone there." }\end{array}$ \\
\hline
\end{tabular}

Figure 2: A example of our qualitative codes, showing body, ritual, and social space representation, part of the "Representation" theme.

the tickets." they related; "The moment in which you...get into the car to go to the show." In complement, accessibility considerations included making audience members feel comfortable while wearing the headset, and supporting different languages for audience needs.

Participants further discussed the importance of social democratization. This included giving VR participants the opportunity to dress up, but not forcing them to do so. One participant revealed that they "rail against the notion of having to dress up to go to the theatre or the opera...simply because I think it just puts an awful lot of people off...there [are] all these kinds of social norms that I certainly didn't grow up with...so everything I've ever done in my life in terms of making work has been...to democratize access."

\subsection{Virtual Representation}

Finally, virtual representation was a key theme, including the representation of performer and audience bodies, the sense of ceremony and occasion, and the social space itself. When discussing body representation, participants exposed the tension between representing performers and audience members as avatars versus capturing gestures and bodies through point clouds. While avatars gave audiences the freedom to represent themselves in any way they choose, participants emphasized the importance of representing human imperfections through "the pudginess of the real world."

Representing the sense of ceremony was likewise important. This included the ceremony of dressing up, intermission, and the pre-and-post social experience. One way to do this was to layer physical and virtual interaction. Comically, a participant remembered that after "doing an online broadcast, which nearly killed us back in fune...the best part of that was imbibing all alcohol that was within my reach afterwards in a Zoom call with about 50 people or going that happened on some level." They elaborated that if it were possible to layer physical and virtual interactions, "you break through social barriers straight away." Conjointly, participants envisioned a virtual representation of the theater physical space and metaphorical "lobby" in VR theatre that did not directly map directly onto the real world.

\section{DISCUSSION}

The goal of this work was to consider a new form of immersive VR opera. Considering how to design immersive opera is inherently disruptive to centuries of tradition. For this reason, the design space of our focus groups was open-ended; we did not limit participants to consider whether an immersive opera should include remote cast members or remote audiences, and if it should be pre-recorded or live-streamed. In the sections below, we consider how to enable an immersive and socially connected opera based on our themes of immersion, agency, democratization, and virtual representation, and imagine such an experience. Notably, while our discussion is contextualized in opera, our findings may generalize to other theater experiences exploring new ways to connect with audiences in the COVID-19 pandemic.

\subsection{Limitations}

We were chiefly limited by the complex form of VR opera. Further, participants were experts in opera and technology, but their perspectives may not have captured those of opera audiences with limited technology exposure.

\subsection{Enabling Immersive Opera}

We found that immersion could evoke audience emotion and transformation, both through sensory experiences and agency. To enable immersion, our findings suggest that the visual form should take advantage of spatial presence, allowing audiences to have a upclose experience of the performance. Further, a new form of opera should use presence to develop transformative narratives that allow audience members to more fully connect with the art, similar to VR conservation experiences that help laypeople appreciate endangered species [23]. To effectively create presence and transformative narratives, we found that it is important to facilitate different forms of agency, as described by Striner [39]. Likewise, it is essential to establish a safe space that moderates trolling behavior. In literature, this has been described as the "magic circle" [38].

\subsection{Enabling Socially Connected Opera}

In complement, democratization and virtual representation were key to enabling a socially connected opera. Democratizing opera consists of creating an intuitive technology onboarding process, and accounting for accessibility needs such as language. Social democratization further considers onboarding and accessibility in context of the immersive social experience; participants wanted audiences to engage with the entire theater experience, from the moment of buying a ticket, dressing up for the performance, experiencing the show, and socializing with others audience members during intermission and after the performance, akin to the social ritual described by Quinn [34]. The virtual representation theme considered how to represent this social experience; participants described different ways of representing audience members, integrating physical interactions into the social virtual experience, and 


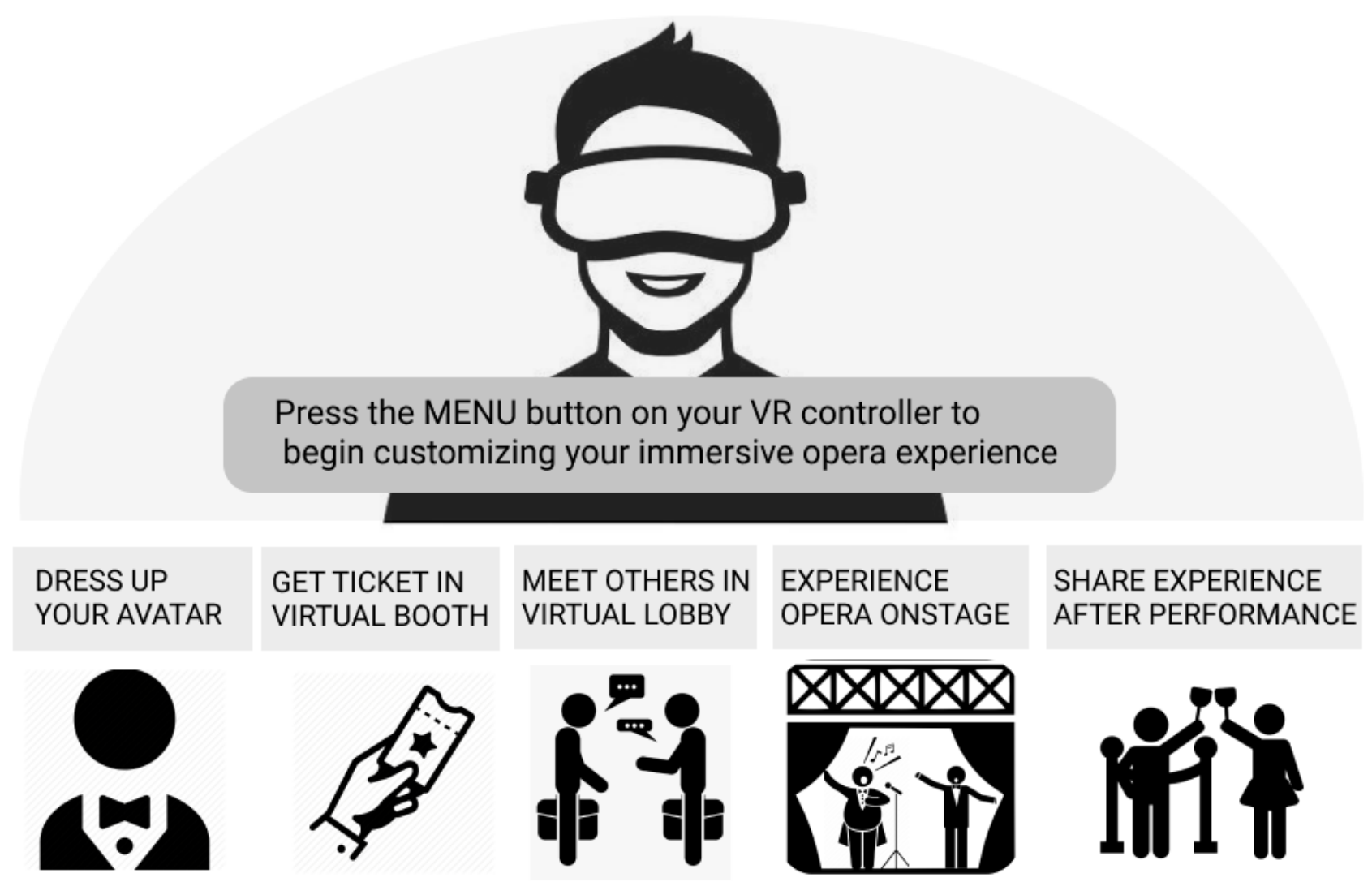

Figure 3: A representation of the opera experience based on our findings. Audience members would first dress up their avatar, then pickup their ticket in a virtual booth. They would meet friends in a virtual lobby before the show, then experience the opera alongside the singers onstage. After the show, audience members would reconnect with friends in a virtual lobby to share their experience.

creating new representations of the theater space and lobby, such as The Under Presents live theater experience [8].

\subsection{Imagining an Immersive VR Opera}

Overall, our findings suggest the need to make social rituals part of the immersive experience. We present an imagined representation of a fully immersive VR opera in figure 3 that includes these rituals. First, audience members would have the opportunity to dress up their virtual representation, then pick their ticket up in a virtual booth. After this, they would have the opportunity to meet friends in a virtual lobby. When the opera begins, audience members would experience the opera onstage, having an intimate experience with the singers. After the show was over, audiences would once again enter the virtual lobby, to reconnect and share their experience with friends.

Some components of this experience aim to recreate opera traditions, including collecting a physical ticket, and celebrating with others after a show. However, such an immersive VR experience also invites audiences beyond the affordances of traditional opera, allowing them to choose how to represent themselves, and to experience opera in a form they would not be able to in a physical space.
Rather than fully replicating the experience of the physical world, our findings provoke designers to ask themselves what real-world experiences they should bring into an immersive context, and what digital affordances can elevate a traditional art into an immersive VR form.

\section{CONCLUSION}

We ran three focus groups to consider how creative, multisensory, and social VR technology may be use to develop a new form of immersive opera. We found that it was important to give audiences agency to interact, to democratize personal and social experiences, and to consider different representations of their bodies, social rituals, and the theater space. Using these findings, we envisioned a new form of immersive VR opera that couples physical traditions with digital affordances.

Future work will consider our findings in context of liveness and remoteness. We will consider if immersive VR opera would be more effective as a pre-recorded experience for audiences to enjoy independently, or as a live-stream, with remote cast members and audiences. In the era of COVID-19, this research supports the 
art form by making it accessible and approachable for modern audiences.

\section{ACKNOWLEDGMENTS}

This work was supported by the European Union's Horizon 2020 Research and Innovation programme under Grant Agreement no. 870610 for the TRACTION project.

\section{REFERENCES}

[1] Virginia Braun and Victoria Clarke. 2006. Using thematic analysis in psychology Qualitative research in psychology 3, 2 (2006), 77-101.

[2] Virginia Braun and Victoria Clarke. 2012. Thematic analysis. (2012)

[3] Jeanne H Brockmyer, Christine M Fox, Kathleen A Curtiss, Evan McBroom, Kimberly M Burkhart, and Jacquelyn N Pidruzny. 2009. The development of the Game Engagement Questionnaire: A measure of engagement in video gameplaying. Journal of Experimental Social Psychology 45, 4 (2009), 624-634.

[4] Teresa Cerratto-Pargman, Chiara Rossitto, and Louise Barkhuus. 2014. Understanding Audience Participation in an Interactive Theater Performance. In Proceedings of the 8th Nordic Conference on Human-Computer Interaction: Fun, Fast, Foundational (Helsinki, Finland) (NordiCHI '14). ACM, New York, NY, USA, 608-617. https://doi.org/10.1145/2639189.2641213

[5] Chia-Chen Chen and Yi-Chen Lin. 2018. What drives live-stream usage intention? The perspectives of flow, entertainment, social interaction, and endorsement. Telematics and Informatics 35, 1 (2018), 293-303.

[6] Mihaly Csikszentmihalyi. 1990. Flow: The psychology of optimal performance.

[7] James J Cummings and Jeremy N Bailenson. 2016. How immersive is enough? A meta-analysis of the effect of immersive technology on user presence. Media Psychology 19, 2 (2016), 272-309.

[8] Jesse Damiani. 2019. 'The Under Presents' Is A Novel Exploration Of VR And Live Immersive Theatre. https://www.forbes.com/sites/jessedamiani/2019/11/19/theunder-presents-is-a-novel-exploration-of-vr-and-live-immersivetheatre/?sh $=798 \mathrm{a} 416 \mathrm{~d} 7455$

[9] Huong Q Dinh, Neff Walker, Larry F Hodges, Chang Song, and Akira Kobayashi. 1999. Evaluating the importance of multi-sensory input on memory and the sense of presence in virtual environments. In Virtual Reality, 1999. Proceedings. IEEE. IEEE, 222-228.

[10] Stefan Engeser and Falko Rheinberg. 2008. Flow, performance and moderators of challenge-skill balance. Motivation and Emotion 32, 3 (2008), 158-172.

[11] Laura Ermi and Frans Mäyrä. 2005. Fundamental components of the gameplay experience: Analysing immersion. Worlds in play: International perspectives on digital games research 37, 2 (2005), 37-53.

[12] Alberto Gallace, Mary K Ngo, John Sulaitis, and Charles Spence. 2012. Multisensory presence in virtual reality: possibilities \& limitations. In Multiple sensorial media advances and applications: New developments in MulSeMedia. IGI Global, $1-38$.

[13] Rosemary Garris, Robert Ahlers, and James E Driskell. 2002. Games, motivation, and learning: A research and practice model. Simulation \& gaming 33, 4 (2002), 441-467.

[14] Gheorghita Ghinea, Christian Timmerer, Weisi Lin, and Stephen R Gulliver. 2014 Mulsemedia: State of the art, perspectives, and challenges. ACM Transactions on Multimedia Computing, Communications, and Applications (TOMM) 11, 1s (2014), 17.

[15] Stephen Gilroy, Julie Porteous, Fred Charles, and Marc Cavazza. 2012. Exploring passive user interaction for adaptive narratives. In Proceedings of the $2012 \mathrm{ACM}$ international conference on Intelligent User Interfaces. ACM, 119-128.

[16] Oliver L. Haimson and John C. Tang. 2017. What Makes Live Events Engaging on Facebook Live, Periscope, and Snapchat. In Proceedings of the 2017 CHI Conference on Human Factors in Computing Systems (Denver, Colorado, USA) (CHI '17). ACM, New York, NY, USA, 48-60. https://doi.org/10.1145/3025453.3025642

[17] Morris B. Holbrook and Elizabeth C. Hirschman. 1982. The Experiential Aspects of Consumption: Consumer Fantasies, Feelings, and Fun. Fournal of Consumer Research 9, 2 (1982), 132. https://doi.org/10.1086/208906

[18] Ali Israr, Siyan Zhao, Kaitlyn Schwalje, Roberta Klatzky, and Jill Lehman. 2014. Feel effects: enriching storytelling with haptic feedback. ACM Transactions on Applied Perception (TAP) 11, 3 (2014), 11

[19] Robert JK Jacob, Audrey Girouard, Leanne M Hirshfield, Michael S Horn, Orit Shaer, Erin Treacy Solovey, and Jamie Zigelbaum. 2008. Reality-based interaction: a framework for post-WIMP interfaces. In Proceedings of the SIGCHI conference on Human factors in computing systems. ACM, 201-210.

[20] Dhruv Jain, Misha Sra, Jingru Guo, Rodrigo Marques, Raymond Wu, Justin Chiu, and Chris Schmandt. 2016. Immersive Scuba Diving Simulator Using Virtual Reality. In Proceedings of the 29th Annual Symposium on User Interface Software and Technology. ACM, 729-739.
[21] Jack Jansen, Shishir Subramanyam, Romain Bouqueau, Gianluca Cernigliaro, Marc Martos Cabré, Fernando Pérez, and Pablo Cesar. 2020. A Pipeline for Multiparty Volumetric Video Conferencing: Transmission of Point Clouds over Low Latency DASH. In Proceedings of the 11th ACM Multimedia Systems Conference (Istanbul, Turkey) (MMSys '20). Association for Computing Machinery, New York, NY, USA, 341-344. https://doi.org/10.1145/3339825.3393578

[22] Kristian Kiili, Timo Lainema, Sara de Freitas, and Sylvester Arnab. 2014. Flow framework for analyzing the quality of educational games. Entertainment Computing 5, 4 (2014), 367-377.

[23] June Kim and Tomasz Bednarz. 2017. Virtual reality to save endangered animals: Many eyes on the wild. In 2017 IEEE Virtual Reality (VR). IEEE, 436-436.

[24] David S. Kirk, David Chatting, Paulina Yurman, and Jo-Anne Bichard. 2016. Ritual Machines I and II. Proceedings of the 2016 CHI Conference on Human Factors in Computing Systems (2016). https://doi.org/10.1145/2858036.2858424

[25] Tuck W. Leong and Peter C. Wright. 2013. Revisiting social practices surrounding music. Proceedings of the SIGCHI Conference on Human Factors in Computing Systems (2013). https://doi.org/10.1145/2470654.2466122

[26] Jie Li, Yiping Kong, Thomas Röggla, Francesca De Simone, Swamy Ananthanarayan, Huib de Ridder, Abdallah El Ali, and Pablo Cesar. 2019. Measuring and Understanding Photo Sharing Experiences in Social Virtual Reality. In Proceedings of the 2019 CHI Conference on Human Factors in Computing Systems (Glasgow, Scotland Uk) (CHI '19). Association for Computing Machinery, New York, NY, USA, 1-14. https://doi.org/10.1145/3290605.3300897

[27] Moira Maguire and Brid Delahunt. 2017. Doing a thematic analysis: A practical, step-by-step guide for learning and teaching scholars. All Ireland fournal of Higher Education 9, 3 (2017).

[28] José Martins, Ramiro Gonçalves, Frederico Branco, Luís Barbosa, Miguel Melo, and Maximino Bessa. 2017. A multisensory virtual experience model for thematic tourism: A Port wine tourism application proposal. fournal of destination marketing \& management 6, 2 (2017), 103-109.

[29] Nora McDonald, Sarita Schoenebeck, and Andrea Forte. 2019. Reliability and inter-rater reliability in qualitative research: Norms and guidelines for CSCW and HCI practice. Proceedings of the ACM on Human-Computer Interaction 3, CSCW (2019), 1-23.

[30] Alison McMahan. 2003. Immersion, engagement and presence. The video game theory reader 67 (2003), 86.

[31] Christopher Morris. 2010. Digital diva: opera on video. The Opera Quarterly 26, 1 (2010), 96-119.

[32] Janet H Murray. 2017. Hamlet on the holodeck: The future of narrative in cyberspace. MIT press.

[33] Marianna Obrist, Carlos Velasco, Chi Vi, Nimesha Ranasinghe, Ali Israr, Adrian Cheok, Charles Spence, and Ponnampalam Gopalakrishnakone. 2016. Sensing the future of $\mathrm{HCI}$ : touch, taste, and smell user interfaces. interactions 23, 5 (2016), 40-49.

[34] Bernadette Quinn. 2003. Symbols, practices and myth-making: cultural perspectives on the Wexford Festival Opera. Tourism Geographies 5, 3 (2003), 329-349. https://doi.org/10.1080/14616680309710

[35] Nimesha Ranasinghe, Pravar Jain, Shienny Karwita, David Tolley, and Ellen YiLuen Do. 2017. Ambiotherm: Enhancing Sense of Presence in Virtual Reality by Simulating Real-World Environmental Conditions. In Proceedings of the 2017 CHI Conference on Human Factors in Computing Systems (Denver, Colorado, USA) (CHI '17). ACM, New York, NY, USA, 1731-1742. https://doi.org/10.1145/3025453. 3025723

[36] David J Shernoff and Mihaly Csikszentmihalyi. 2009. Cultivating engaged learners and optimal learning environments. Handbook of positive psychology in schools (2009), 131-145.

[37] Thomas Smith, Marianna Obrist, and Peter Wright. 2013. Live-streaming Changes the (Video) Game. In Proceedings of the 11th European Conference on Interactive TV and Video (Como, Italy) (EuroITV '13). ACM, New York, NY, USA, 131-138. https://doi.org/10.1145/2465958.2465971

[38] Jaakko Stenros. 2014. In defence of a magic circle: the social, mental and cultural boundaries of play. Transactions of the Digital Games Research Association 1, 2 (2014).

[39] Alina Striner, Sasha Azad, and Chris Martens. 2019. A Spectrum of Audience Interactivity for Entertainment Domains. In Proceedings of the 2019 International Conference on Interactive Digital Storytelling (ICIDS).

[40] Tynan Sylvester. 2013. Designing games: A guide to engineering experiences. " O’Reilly Media, Inc.".

[41] John C. Tang, Gina Venolia, and Kori M. Inkpen. 2016. Meerkat and Periscope: I Stream, You Stream, Apps Stream for Live Streams. In Proceedings of the 2016 CHI Conference on Human Factors in Computing Systems (San Jose, California, USA) (CHI '16). ACM, New York, NY, USA, 4770-4780. https://doi.org/10.1145/ 2858036.2858374

[42] Ben Walmsley. 2011. Why people go to the theatre: A qualitative study of audience motivation. Fournal of Customer Behaviour 10, 4 (2011), 335-351. https: //doi.org/10.1362/147539211x13210329822545

[43] Donghee Yvette Wohn and Guo Freeman. 2019. Live Streaming, Playing, and Money Spending Behaviors in eSports. Games and Culture 1 (2019), 16. 
[44] Tim Wulf, Frank M Schneider, and Stefan Beckert. 2020. Watching players: An exploration of media enjoyment on Twitch. Games and culture 15, 3 (2020),

$328-346$. 